\title{
Tuberculous Spondylitis and Coincidental Spinal Multiple Myeloma: A Case Report
}

\section{Prinsen SD ${ }^{1 *}$, Groot $D^{2}$, Oude Lashof $\mathrm{AML}^{3}$ and Willems PC4}

${ }^{1}$ Department of Orthopaedics, Maastricht University Medical Center, The Netherlands

${ }^{2}$ Department of Orthopaedics, Sint Maartenskliniek Woerden, The Netherlands

${ }^{3}$ Department of Medical Microbiology and Internal Medicine, School for Public Health and Primary Care (CAPHRI), Maastricht University Medical Center, The Netherlands ${ }^{4}$ Department of Orthopaedics, School for Public Health and Primary Care (CAPHRI), Maastricht University Medical Center, The Netherlands

\section{Abstract}

Background content: Tuberculous spondylitis (Pott's disease) is known for nonspecific findings which can mimic metastatic disease. Spinal pain, local tenderness and night sweats may be presenting symptoms. There is no pathognomic radiographic appearance of tuberculous spondylitis.

Back pain may also be the first sign of multiple myeloma (MM) and is usually caused by bone destruction or vertebral collapse. The tumour itself can also cause pain by nerve or spinal cord damage resulting from direct pressure or secondary peripheral neuropathies. $\mathrm{MM}$ is often complicated by infectious diseases caused by the mainly cell mediated and humoral immune deficiency.

Purpose: To report a case of a patient who presented with Pott's disease and multiple myeloma in the same vertebra.

Study Design: Case Report

Patient Sample: Description of one case

Outcome measures: Multiple myeloma and Pott's disease can coincide. If one of these diseases is overlooked, it may have serious consequences for the patient.

Methods: We present a case of a patient with Pott's disease and multiple myeloma in the same vertebra. This is shown on radiographic findings, laboratory results and pathologic findings.

Results: Radiographic findings, laboratory results and pathologic findings showed a Pott's disease and multiple myeloma in the same vertebra.

Conclusion: We present a patient with persisting back pain, who was initially diagnosed with spinal tuberculosis Despite antituberculous medication he developed signs of spinal cord compression and was operated on. Retrieved tissue samples revealed multiple myeloma.

This case highlights that, as MM may predispose for active TB, the two diagnoses may coincide. Because of similarity of clinical appearance one of them may be overlooked, which may have serious consequences for the patient.

\section{Case Report}

In December 2009, a 68-year-old Caucasian male visited the orthopaedic outpatient clinic with complaints of disabling back pain, weight loss of six kilograms, and night sweating. The pain was located on the midthoracic level and radiated to the front of the thorax. There were no symptoms of bladder or bowel dysfunction. Coughing and pressing did not aggravate the pain. Non Steroidal Anti Inflammatory Drugs did not relieve pain. He was known with benign prostrate hypertrophy. There was no past medical or family history of tuberculosis (TB) and he had never visited endemic areas of TB.

Physical examination revealed a mild thoracic kyphoscoliosis with local tenderness over the spinous processes T4-T6. Bilateral compression of the thorax was not painful. The straight leg raise test was negative and there were no signs of neurological deficit.

Plain radiographs of the thoracic spine showed disc space narrowing and spondylosteophytes, and signs of a collapsed vertebral body of T5 (Figure 1). An MRI of the spine showed the collapsed body of T5 with paravertebral fluid collections. There was a normal aspect of the adjacent intervertebral discs T4-T5 and T5-T6 (Figures 2 and 3).

Laboratory findings showed an elevated sedimentation rate (50 $\mathrm{mm} / \mathrm{h})$, anemia $(6.3 \mathrm{mmol} / \mathrm{l})$, leucocytes $4.6 \times 10^{\wedge} 9 / 1$, CRP $47 \mathrm{mg} / \mathrm{L}$ and a creatinine of $202 \mu \mathrm{mol} / \mathrm{L}$ (Table 1 ).

The patient was admitted for a CT-guided biopsy of the collapsed T5 body. Cultures and pathological examination were negative, however Ziehl-Neelsen staining and Polymerase Chain Reaction (PCR) for Mycobacterium tuberculosis were positive. A CT-scan of the thorax showed signs of pulmonary TB. PCR on sputum was also positive for M. tuberculosis.

\begin{tabular}{|l|c|c|c|c|c|}
\hline (Weeks) & Admittance & $\mathbf{+ 4}$ & $\mathbf{+ 6}$ & $\mathbf{+ 8}$ & $\mathbf{+ 2 0}$ \\
\hline Sedimentation rate & 50 & 54 & 66 & 14 & 6 \\
\hline Hb & 6.3 & 5.9 & 5.5 & 5.8 & 5.2 \\
\hline Leucocytes & 4.6 & 6.7 & 8.7 & 8.7 & 1.5 \\
\hline CRP & 47 & 47 & 243 & 41 & 9.1 \\
\hline Creatinine & 202 & 282 & 454 & 415 & 338 \\
\hline
\end{tabular}

Table 1: Development of laboratory findings in time.

*Corresponding author: Prinsen SD, Department of Orthopaedics, Maastricht University Medical Center, The Netherlands, Tel: 3143388 2222; E-mail: sandraprinsen04@hotmail.com

Received November 13, 2013; Accepted December 10, 2013; Published December 12, 2013

Citation: Prinsen SD, Groot D, Oude Lashof AML, Willems PC (2013) Tuberculous Spondylitis and Coincidental Spinal Multiple Myeloma: A Case Report. J Spine 2 148. doi:10.4172/2165-7939.1000148

Copyright: (c 2013 Prinsen SD, et al. This is an open-access article distributed under the terms of the Creative Commons Attribution License, which permits unrestricted use, distribution, and reproduction in any medium, provided the original author and source are credited. 


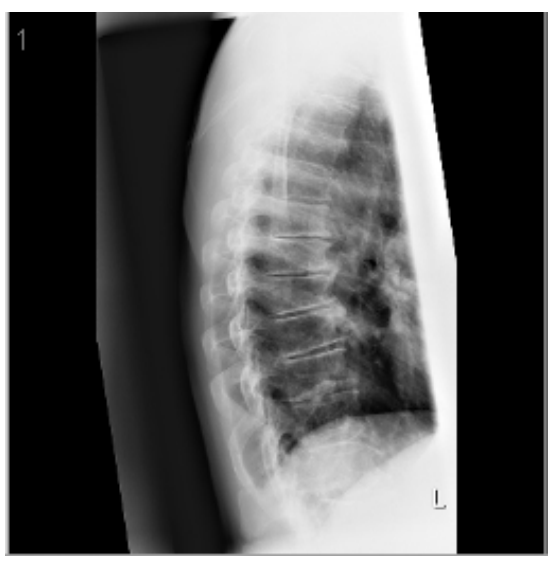

Figure 1: Lateral view of thoracic spine: disc space narrowing and spondylosteophytes, and signs of a collapsed vertebral body of T5.

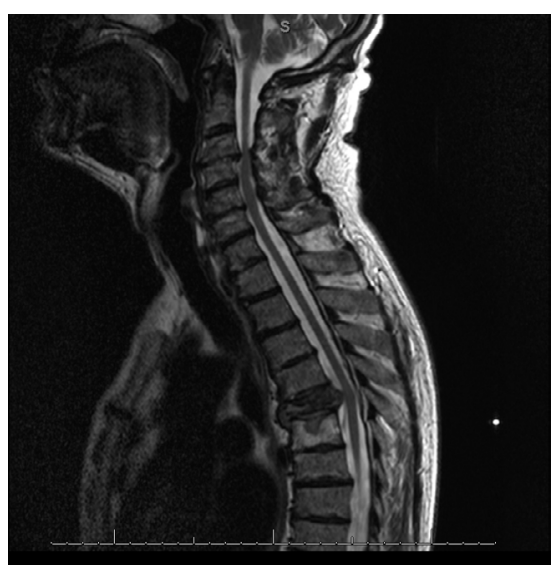

Figure 2: Sagittal view of the thoracic spine (MRI)

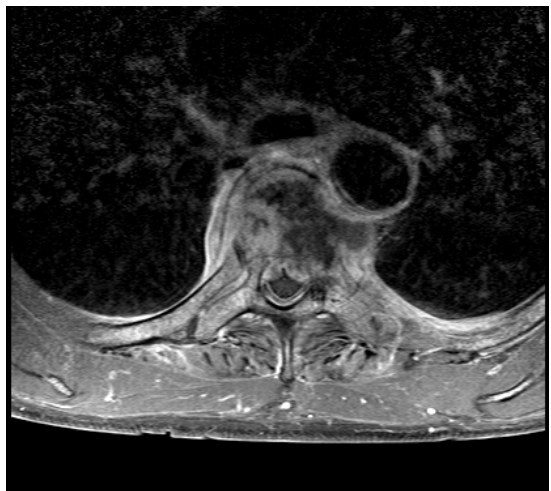

Figure 3: Transverse view of the vertebral body of T5 (MRI)

Treatment with pyrazinamide (2000 mg daily), ethambutol (1600 $\mathrm{mg}$ daily), rifampicin (600 $\mathrm{mg}$ daily) and isoniazide (300 $\mathrm{mg}$ daily) was started in December 2009.

Three weeks after the onset of the TB medication, our patient developed loss of sensibility in the lower legs. A MRI-scan showed a severely narrowed spinal canal with encroachment of tumorous tissue and fluid compressing the spinal cord. Emergency decompression by laminectomy and partial corporectomy of T5 was performed. For stabilisation, a posterior instrumented fusion from T3 to T8 with cage interposition at T5. (CD Legacy+Pyramesh, Medtronic, Memphis, USA) was executed (Figures $4 \mathrm{a}$ and $4 \mathrm{~b}$ ).

Retrieved T5 vertebral body tissue samples showed a plasma cell accumulation (plasmocytoma, Figure 5) and a positive $3 \mathrm{~cd} 138$ stain (Figure 6). In serum IgG was elevated with free lambda light chains $(2140 \mathrm{mg} / \mathrm{l})$. The haematological oncologist was consulted for treatment of this multiple myeloma.

Postoperatively, the neurological status of the patient recovered completely. The patient was mobilised with a thoracolumbar orthosis. After fourteen days a superficial wound infection with Escherichia coli developed, which was treated initially with Amoxicillin/clavulanic acid.

Because of persistent wound infection, exploration and debridement of the wound was performed three weeks after the index surgery and 60 gentamycine beads were left in situ. Wound healing was uneventful and at the 60th day postoperative and laboratory findings had returned to

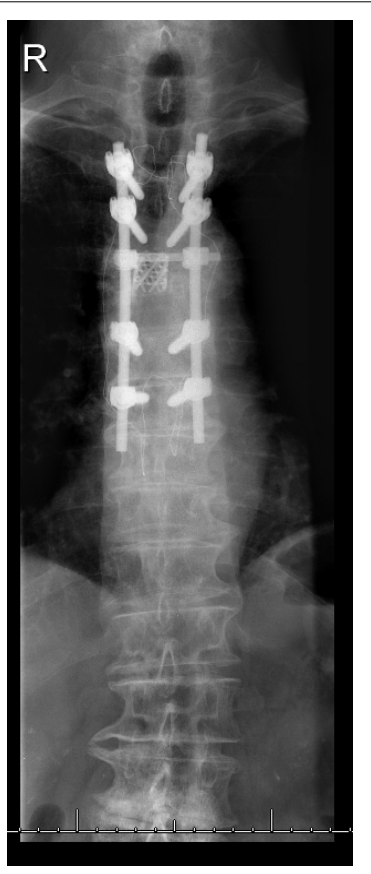

Figure 4a: AP view after stabilisation.

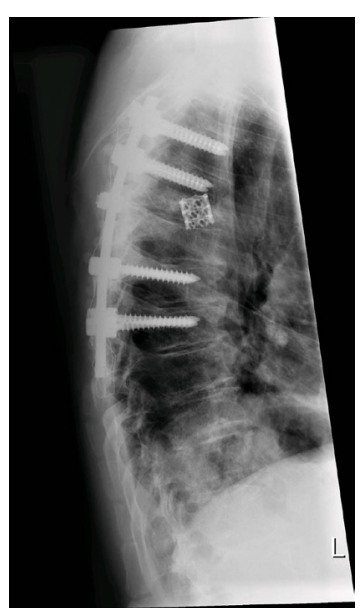

Figure 4b: Lateral view after stabilisation. 


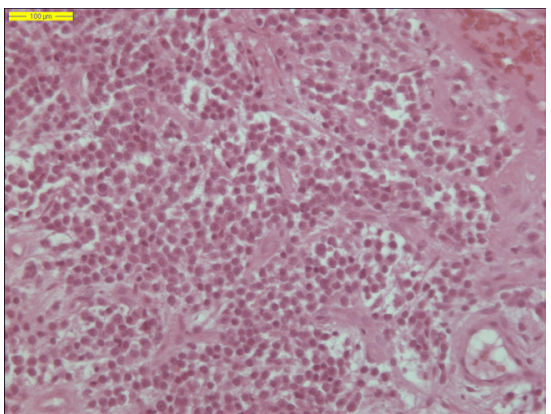

Figure 5: Pathology: a plasma cell accumulation.

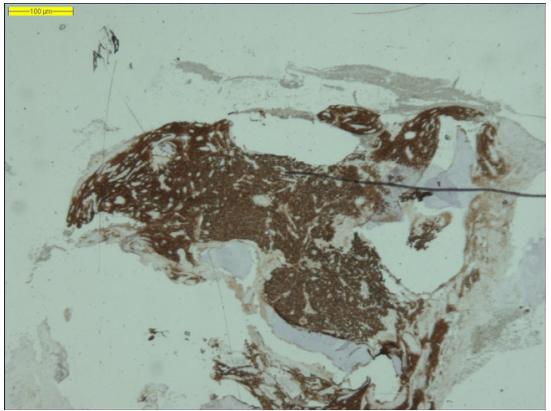

Figure 6: Pathology: a positive $3 \mathrm{~cd} 138$ stain

normal. The patient was discharged and treated for six months with TB medication ( 2 months quadruple therapy, 4 months triple therapy). The MM was treated with a schedule of $9 \mathrm{mg} / \mathrm{m}^{2}$ adriamycine one time and thalidomide $200 \mathrm{mg}$ daily. Due to the wound infection, the prednisone was taken out to maintain wound healing.

\section{Discussion}

Spontaneous infective discitis is a recognized cause of back pain in adults [1]. Common presenting features are persistent backache, fever and raised ESR. In patients with immunosuppression, e.g. leukemia, chemotherapy treatment, organ transplantation or diabetes mellitus, spontaneous discitis has been reported to be more prevalent [1]. Unlike in postoperative discitis, which is mainly caused by $S$. aureus or $S$. epidermidis, patients with spontaneous discitis show a wide variety of Gram positive, Gram negative, mycobacterial, and fungal organisms [2].

TB spondylitis (Pott's disease) is known for atypical findings which can be similar to manifestation of metastatic malignant disease [3]. Spinal pain, local tenderness and night sweats may be presenting symptoms. There is no specific radiographic finding pathognomic for tuberculous spondylitis [4]. The extent of bone involvement is frequently underestimated on plain X-rays. Only loss of endplate definition and slight disc space narrowing can be seen. Most distinguishing for spinal $\mathrm{TB}$ is the relative sparing of intervertebral discs. This can be explained by the fact that Mycobacterium species lack the proteolytic enzyme that is responsible for destruction of the disc. Another characteristic finding for Pott's disease is the formation of paravertebral abscesses which can be located anterior, posterior, lateral and circumferential. The probability of a tuberculous spondylitis increases when the abscess is large [5]. The diagnosis should be confirmed by culture of needle biopsy aspirate.

Multiple myeloma (also known as Kahler's disease) is a malignant proliferation of plasma cells derived from a single clone. Bone pain is the most common symptom of the disease, affecting nearly $70 \%$ of the patients [5,6]. Unlike the pain in metastatic disease, which often is worse at night, the pain of myeloma is precipitated by movement. The bony lesions which occur in MM are caused by the proliferation of tumour cells and activation of osteoclasts that destroy the bone. The bone lesions are lytic in nature and are rarely associated with osteoblastic new bone formation. Therefore, a bone scintigraphy is less useful for diagnosis compared to plain radiography. Laboratorial characteristics of multiple myeloma are a high serum calcium level caused by the osteolysis, renal failure due to the high excretion of light chains and deposition into the kidneys. Deficient antibody production, abnormal cell-mediated immunity, and - in advanced state - neutropenia predispose for infections. Chemotherapy induced myelosuppression further increase the propensity to infection.

In the Netherlands there has been an increase in the incidence of bone and joint tuberculosis in the past decade [7]. In the literature we found only one report of MM complicated by tuberculous spondylitis. To our knowledge, this is the first case report of tuberculous spondylitis as the primary manifestation of multiple myeloma, and we conclude that in patients with tuberculous spondylitis without improvement of symptoms on appropriate antituberculous medication, there should be a high clinical suspicion of underlying immunosuppressing disease. In view of the similarities in the clinical features of tuberculous spondylitis and MM, awareness that both diagnoses may coincide is warranted, as earlier instalment of appropriate treatment will usually lead to a better outcome of the disease and may avoid severe complications.

\section{References}

1. Honan M, White GW, Eisenberg GM (1996) Spontaneous infectious discitis in adults. Am J Med 100: 85-89.

2. Tali ET (2004) Spinal infections. Eur J Radiol 50: 120-133.

3. Ringshausen FC, Tannapfel A, Nicolas V, Weber A, Duchna HW, et al. (2009) A fatal case of spinal tuberculosis mistaken for metastatic lung cancer: recalling ancient Pott's disease. Ann Clin Microbiol Antimicrob 8: 32

4. Joseffer SS, Cooper PR (2005) Modern imaging of spinal tuberculosis. J Neurosurg Spine 2: 145-150.

5. Burton CH, Fairham SA, Millet B, DasGupta R, Sivakumaran M (1998) Unusua aetiology of persistent back pain in a patient with multiple myeloma: infectious discitis. J Clin Pathol 51: 633-634.

6. Kalambokis GN, Christou L, Tsianos EV (2009) Multiple myeloma presenting with an acute bacterial infection. Int J Lab Hematol 31: 375-383.

7. Jutte PC, van Loenhout-Rooyackers JH, Borgdorff MW, van Horn JR (2004) Increase of bone and joint tuberculosis in The Netherlands. J Bone Joint Surg $\mathrm{Br}$ 86: 901-904. 